\title{
National scale-up of tuberculosis-human immunodeficiency virus collaborative activities in Myanmar from 2005 to 2016 and tuberculosis treatment outcomes for patients with human immunodeficiency virus-positive tuberculosis in the Mandalay Region in 2015
}

\author{
Phyo Theingi ${ }^{a, *}$, Anthony D. Harries ${ }^{b, c}$, Khin Thet Wai ${ }^{d}$, Hemant D. Shewade ${ }^{b, e}$, Saw Saw ${ }^{d}$, Than Win ${ }^{f}$, Saw Thein ${ }^{a}$, \\ Myo Su Kyi ${ }^{a}$, Htun Nyunt $\mathrm{Oo}^{\mathrm{f}}$ and Si Thu Aung ${ }^{\mathrm{a}}$
}

${ }^{a}$ National Tuberculosis Programme, Department of Public Health, Ministry of Health and Sports, Nay Pyi Taw, Myanmar; ${ }^{b}$ International Union Against Tuberculosis and Lung Disease, Paris, France; ${ }^{C}$ Faculty of Infectious Diseases and Tropical Medicine, London School of Hygiene and Tropical Medicine, London, UK; ${ }^{d}$ Department of Medical Research, Ministry of Health and Sports, Myanmar; International Union Against Tuberculosis and Lung Disease (The Union), South-East Asia Office, New Delhi, India; ${ }^{f_{N}}$ ational AIDS Programme,

Department of Public Health, Ministry of Health and Sports, Myanmar

*Corresponding author: E-mail: dr.phyotheingi12@gmail.com

Received 22 June 2017; revised 3 November 2017; editorial decision 13 November 2017; accepted 16 November 2017

\begin{abstract}
Background: HIV-associated TB is a serious public health problem in Myanmar. Study objectives were to describe national scale-up of collaborative activities to reduce the double burden of TB and HIV from 2005 to 2016 and to describe TB treatment outcomes of individuals registered with HIV-associated TB in 2015 in the Mandalay Region.

Methods: Secondary analysis of national aggregate data and, for treatment outcomes, a cohort study of patients with HIV-associated TB in the Mandalay Region.

Results: The number of townships implementing collaborative activities increased from 7 to 330 by 2016 . The number of registered TB patients increased from 1577 to 139625 in 2016, with the number of individuals tested for HIV increasing from 432 to 114180 (82\%) in 2016: 10971 (10\%) were diagnosed as HIV positive. Uptake of co-trimoxazole preventive therapy (CPT) and antiretroviral therapy (ART) nationally in 2016 was $77 \%$ and $52 \%$, respectively. In the Mandalay Region, treatment success was $77 \%$ and mortality was $18 \%$ in 815 HIV-associated TB patients. Risk factors for unfavourable outcomes and death were older age ( $\geq 45$ years) and not taking CPT and/or ART.
\end{abstract}

Conclusion: Myanmar is making good progress with reducing the HIV burden in TB patients, but better implementation is needed to reach 100\% HIV testing and 100\% CPT and ART uptake in TB-HIV co-infected patients.

Keywords: Antiretroviral therapy, Co-trimoxazole preventive therapy, HIV, Mandalay, Myanmar, Tuberculosis

\section{Introduction}

Despite steady progress in the implementation of the global Directly Observed Treatment, Short-Course (DOTS) and subsequently the Stop TB strategy since 1995, TB remains an enormous global public health challenge. In 2015, the total number of new cases globally was estimated at 10.4 million and the number of deaths due to TB was estimated at 1.8 million. ${ }^{1}$ In the same year, about 1.2 million people worldwide (11\% of all new TB cases) were thought to develop HIV-associated TB and there were 400000 HIV-associated TB deaths (22\% of all TB deaths). ${ }^{1}$ While the African Region is the one most affected by HIV-associated TB, the South-East Asian Region and the Western Pacific together account for $22 \%$ of the global HIV-associated TB patients, with India, Indonesia, Myanmar, Thailand, China and Vietnam comprising the majority. ${ }^{1}$

HIV adversely affects TB control efforts. Not only is HIV associated with large increases in TB cases, but it also leads to

(c) The Author(s) 2018. Published by Oxford University Press on behalf of Royal Society of Tropical Medicine and Hygiene. All rights reserved. For permissions, please e-mail: journals.permissions@oup.com. 
increased mortality during and after anti-TB treatment. ${ }^{2}$ In 2004 the WHO launched an interim policy document on TB-HIV collaborative activities $^{3}$ and followed this in 2012 with an updated policy that consolidated evidence about the efficacy and effectiveness of HIV-associated TB interventions from randomized controlled trials, observational studies, operational research and best practices from programme implementation. ${ }^{4}$ To reduce the burden of HIV in TB patients, the key interventions are HIV testing and ensuring that HIV-infected TB patients are started on co-trimoxazole preventive therapy (CPT) and antiretroviral therapy (ART) as soon as possible after starting anti-TB treatment. In HIV-infected TB patients, CPT is associated with a $25-46 \%$ mortality reduction and ART with a $64-95 \%$ mortality reduction, with a synergistic effect being observed when the two interventions are combined. ${ }^{5}$

Myanmar is one of the 30 most HIV-associated TB high burden countries in the world, ${ }^{1}$ and in 2014 a sero-sentinel surveillance study documented an HIV prevalence in TB patients of 8.5\%. ${ }^{6}$ Following the 2004 WHO interim policy, TB-HIV collaborative activities in the country started in 2005 in seven townships. The focus of these activities was to reduce the burden of HIV in TB, and the activities have gradually expanded to the whole country of 330 townships. It is important for the national TB and AIDS programmes to obtain a countrywide picture of the increase in townships adopting activities to reduce the burden of HIV in TB, including the numbers and proportions of registered TB patients being tested for HIV and for those who are HIV positive, the numbers and proportions starting on CPT and ART. In 2010 there was a report showing good HIV testing rates and uptake of ART in the integrated HIV care programme for TB patients in the Mandalay Region and presenting generally good outcome results. ${ }^{7}$

With the 2012 updated WHO policy re-emphasizing the need for CPT and ART in all HIV-associated TB patients, with the latter started as soon as possible after initiating TB treatment regardless of the CD4 cell count, it is also important to know how well these interventions are currently being implemented in Myanmar and whether they have any beneficial effect on TB-specific treatment outcomes. It is also important to know whether there are other factors associated with poor treatment outcomes, e.g., gender, older age (as a result of comorbidities) and types and categories of TB, that can be addressed with specific interventions. The objectives of this study therefore were to describe at the national level the scale-up and implementation of TB-HIV collaborative activities to reduce the burden of HIV in TB patients at the township level and in registered TB patients in Myanmar between 2005 and 2016 and to describe the TB treatment outcomes of individual patients registered with HIV-associated TB in the Mandalay Region in 2015.

\section{Materials and methods}

\section{Study design}

For the national study on scale-up of TB-HIV collaborative activities, this was a secondary analysis of national aggregate data on townships and patients registered with TB. For the TB treatment outcome study, this was a cohort study using secondary data of individual patients registered with HIV-associated TB.

\section{Study setting}

General

Myanmar is situated in South-East Asia and has an estimated population of slightly more than 51 million, 70\% of whom live in rural areas, according to the Myanmar National Census conducted in $2014^{8}$ There are 135 ethnic groups, most of whom are poor, with low levels of education. The country is divided administratively into 15 states/regions, 74 districts and 330 townships.

There is a National TB Program (NTP) that, together with partners, offers free-of-charge diagnostic, treatment and followup services for patients with presumptive and confirmed TB, based on the WHO 2010 TB treatment guidelines. ${ }^{9}$ There is also a National AIDS Program (NAP) that, together with partners, offers HIV testing and counselling, and for those found to be HIV positive, care, CPT and ART according to criteria that are in operation at that time. ${ }^{10,11}$ For people living with HIV, TB screening is also regularly carried out: those identified with presumptive TB are referred to the NTP for further investigation while those with no TB-related symptoms are considered for isoniazid preventive therapy provided there are no contraindications.

From 2005, TB and HIV/AIDS control activities were mainly supported by the government along with a number of bilateral international donors. In 2011, Myanmar received financial support from the Global Fund Against AIDS, Tuberculosis and Malaria (GFATM) in two phases (2011-2012 and 2013-2015). During the second phase, the GFATM launched a new funding model and provided performance-based funding that included strong support for the scaling up of TB-HIV collaborative activities in order to assist the country in trying to achieve $100 \% \mathrm{HIV}$ testing uptake and $100 \%$ uptake of CPT and ART in co-infected patients and in meeting or exceeding the global target for HIVassociated TB treatment success at 75\%. ${ }^{4,12}$

\section{TB-HIV collaborative activities at the township level}

Every township has a TB centre that offers sputum smear microscopy for patients presenting with presumptive TB. Those found to be smear-positive are diagnosed as smear-positive pulmonary TB (PTB). For those found to be smear-negative, a clinical and radiographic algorithm for diagnosing smear negative pulmonary TB or extrapulmonary TB (EPTB) is applied. The Xpert MTB/RIF (Mycobacterium tuberculosis/resistance to rifampicin) assay is used in those known to be HIV infected. Patients diagnosed with TB are registered and started on standardized anti-TB treatment that is in line with the WHO TB treatment guidelines. ${ }^{9}$

All registered TB patients are offered HIV testing using rapid diagnostic tests with Determine (AlereDetermine HIV-1/2, Alere International, Galway, Ireland), UniGold (Trinity Biotech, Wicklow, Ireland) and STAT-PAK (Chembio Diagnostic Systems, Medford, NY, USA). Initially, HIV testing was done by NAP staff, but since 2014, HIV testing has been undertaken by NTP staff. HIV-positive TB patients are offered immediate CPT, which was provided first through the NTP staff up to 2014 and since then from the NAP staff for the duration of TB treatment. From 2005, HIV-infected TB patients were offered ART through the NAP staff only if their CD4 lymphocyte count was $<200$ cells $/ \mathrm{mm}^{3}$. From 2012, the criteria for starting ART changed: HIV-infected TB patients were offered 
ART if their CD4 lymphocyte count was $<500$ cells $/ \mathrm{mm}^{3}$. In 2015 , this changed again, with recommendations that all HIV-infected TB patients start ART regardless of their CD4 count. ${ }^{13}$ Again, ART is always offered and managed by NAP staff. The ART regimen from 2005 was a stavudine-based regimen, but in 2015 this changed to a tenofovir-based regimen.

All TB patients are monitored using paper-based treatment cards and registers using standardized definitions for case finding and treatment outcomes. ${ }^{9}$ HIV testing and HIV results are recorded in the township TB centre laboratory register and TB treatment register, with the latter also recording whether CPT and/or ART has been initiated. The data from each township are sent on a quarterly basis to the regional centres, where they are collated and sent on to the national programme for further collation into national quarterly reports. These in turn form the basis of national annual reports.

\section{Townships and study population}

For the national study, the units for assessment were townships implementing TB-HIV collaborative activities and all patients registered with TB in Myanmar between 2005 and 2016. For the individual patient study, the population included all patients registered with HIV-associated TB in the 28 townships in the Mandalay Region between January and December 2015.

\section{Data variables, sources of data and data collection}

For the national study, data variables included year, townships implementing TB-HIV collaborative activities, number of TB patients registered each year in the townships and numbers of patients HIV tested, HIV positive, placed on CPT and started on ART. Sources of data were the national annual TB reports from 2005 to 2015 and the NTP electronic database for 2016.

For the individual patient study in the Mandalay Region, data variables included year, TB registration number, name of the township, gender, age, category of TB (new, relapse, treatment after loss to follow-up, treatment after failure, other previously treated), type of TB (pulmonary and extrapulmonary), started on CPT, started on ART and TB treatment outcomes (cured, completed treatment, loss to follow-up, died, failed, not evaluated [transferred out] and moved to second-line treatment). Treatment success was defined as cured plus treatment completed and unfavourable treatment outcomes were defined as everything else. The source of data was the NTP electronic database file, the NAP-ART register and the Union electronic database file for the Mandalay Region for 2015.

\section{Analysis and statistics}

Aggregate nationwide data for Myanmar were extracted from the annual TB reports and NTP electronic database into an Excel file (Microsoft, Redmond, WA, USA). Similarly, individual patient data for the Mandalay Region were extracted from the electronic databases and the NAP-ART register into another Excel file. For the national data, frequencies and proportions were described and analysed. For individual patient data, demographic and clinical characteristics related to unfavourable treatment outcomes or death were compared using the $\chi^{2}$ test with results presented as RRs and 95\% CIs. Adjusted RRs (with 95\% CIs) were calculated by plotting a multivariable predictive model (log binomial for unfavourable treatment outcomes and modified Poisson for death). Levels of significance were set at $5 \%(p<0.05)$.

\section{Ethics}

Permission to use the secondary data for this study was obtained from the NTP and NAP.

\section{Results \\ National aggregate data}

The number of townships in Myanmar implementing TB-HIV collaborative activities between 2005 and 2016 is shown in Figure 1. Initially there were just seven townships, and this increased gradually to 30 by 2013. In 2014 there was a rapid expansion and all townships in the country were implementing TB-HIV collaborative activities by 2016.

The annual number of registered TB patients in these townships, along with those knowing their HIV status and HIV positivity between 2005 and 2016, are shown in Figure 2. Initially there were just 1577 registered patients, with the number gradually increasing to 13718 in 2012 . Thereafter there was rapid expansion, with the registered number of TB patients increasing to 139625 in 2016. The proportion of patients with known HIV status (newly tested or HIV result already known) varied from year to year, but in the years 2013-2016 it increased progressively (2013, 44\%; 2014, 60\%; 2015, 76\%; 2016, 82\%). HIV positivity also varied from year to year, but in the 3 years from 2014 to 2016, the proportion diagnosed as HIV positive was fairly stable at between 9 and 11\%.

Annual uptake of CPT and ART among TB-HIV co-infected patients is shown in Figures 3 and 4. For CPT, there were no data pertaining just to the townships for 2008-2010. There was variable uptake in the early years when the number of patients was low, but from 2013 to 2016 uptake was between 72 and 89\%. For ART, annual uptake was between 60 and $98 \%$ for 2008-2011. From 2013 onwards, the total numbers accessing

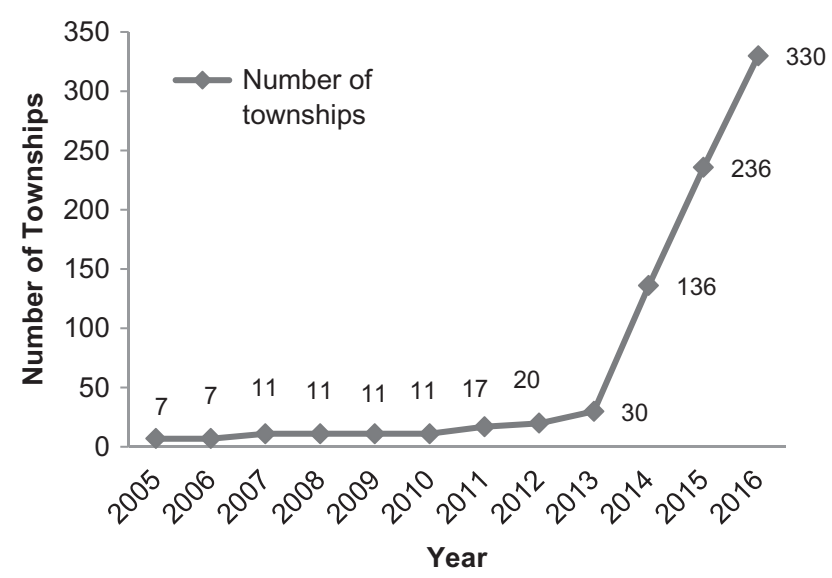

Figure 1. Townships implementing TB-HIV collaborative activities in Myanmar, 2005-2016. 


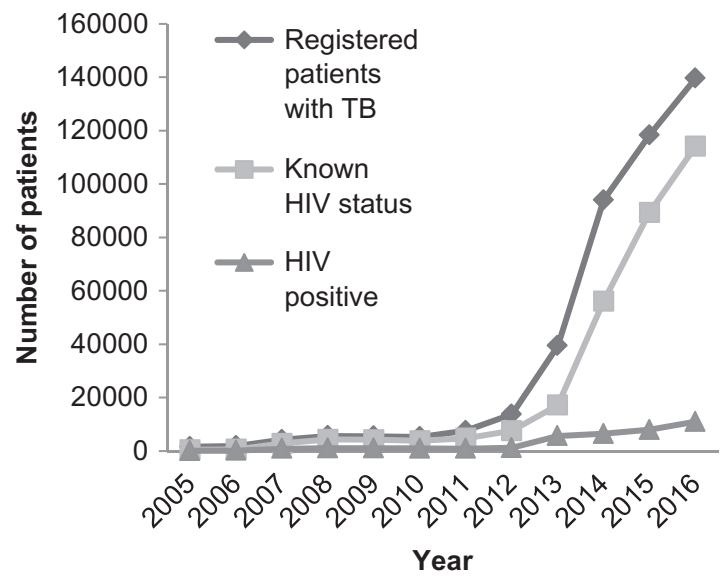

Figure 2. Annual registered patients with TB with known HIV status and HIV positivity in townships implementing TB-HIV collaborative activities in Myanmar, 2005-2016.

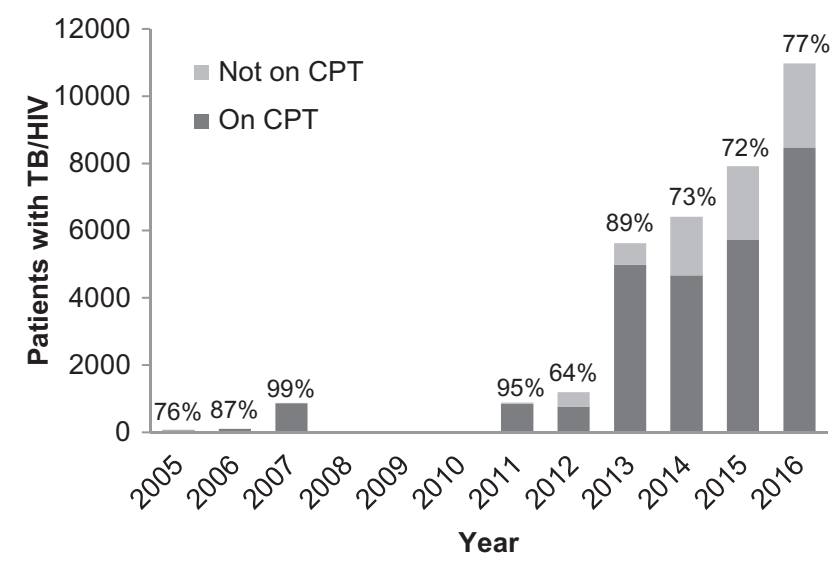

Figure 3. Annual uptake of co-trimoxazole preventive therapy in patients with HIV-associated TB in townships implementing TB-HIV collaborative activities in Myanmar, 2005-2016. CPT=co-trimoxazole preventive therapy; \%=percentage of patients with HIV-associated TB on CPT. There were no specific township data available for CPT uptake for 2008-2010.

ART increased but the proportion of patients on ART varied, though it had increased to $52 \%$ in 2016 .

\section{Regional individual data}

The characteristics of 815 TB-HIV co-infected patients registered in the Mandalay Region in 2015 are shown in Table 1. Most were 15-44 years of age, two-thirds were male and the majority had new pulmonary TB. Nearly three-quarters of the patients were on CPT and ART. Treatment outcomes for the entire cohort are shown in Table 2. Treatment success was 77\%, with the main cause of an unfavourable treatment outcome in the remaining 23\% being death. Characteristics associated with an unfavourable treatment outcome or with death are shown in Tables 3 and 4. The main factors significantly associated with an increased risk of unfavourable treatment outcome and/or death were age $\geq 45$ years, taking CPT alone (significant only for

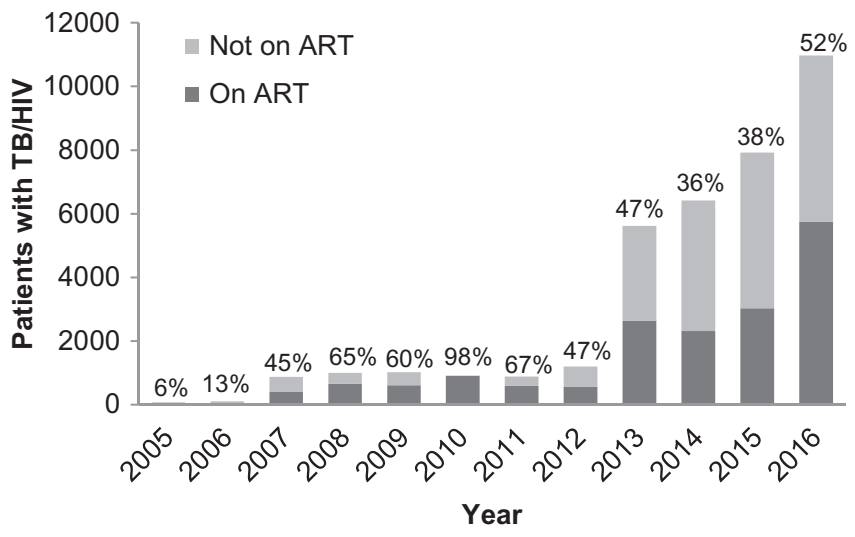

Figure 4. Annual uptake of ART in patients with HIV-associated TB in townships implementing TB-HIV collaborative activities in Myanmar, 2005-2016. ART=antiretroviral therapy; \%=percentage of patients with HIV-associated TB on ART.

Table 1. Demographic and clinical characteristics of patients registered with HIV-associated TB in the Mandalay Region, Myanmar, 2015

Characteristics n (\%)

Total $815(100)$

Age group in years

$\leq 14$

15-44

$606(74)$

$\geq 45$

146 (18)

Gender

Male

Female

Category of TB

New

$718(88)$

Relapse

Treatment after LTFU

Treatment after failure

Type of TB

Pulmonary

Extrapulmonary

Adjunctive therapy

CPT alone

$162(20)$

ART alone

CPT and ART together

No adjunctive treatment

No information

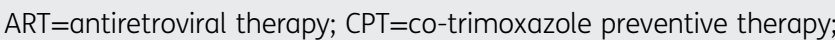
LTFU=loss to follow-up.

death), taking no adjunctive therapy at all or having no details recorded of adjunctive therapy (significant only for death) compared with the reference values. 


\section{Discussion}

This is the first study in Myanmar to describe the scale-up of TB-HIV collaborative activities at the national level between 2005 and 2016 and to assess at the individual level how HIV

Table 2. Treatment outcomes of patients registered with HIVassociated TB in the Mandalay Region, Myanmar, 2015

\begin{tabular}{lc} 
Treatment outcomes & $\mathrm{n}(\%)$ \\
\hline Total enrolled for treatment & $815(100)$ \\
Treatment success & $624(77)$ \\
Cured & $295(36)$ \\
Treatment completed & $329(41)$ \\
Unfavourable treatment outcomes & $191(23)$ \\
Loss to follow-up & $18(2)$ \\
Died & $146(18)$ \\
Failed & $9(1)$ \\
Not evaluated (transferred out) & $11(1)$ \\
Changed to second-line treatment & $7(1)$
\end{tabular}

care and treatment for HIV-associated TB patients related to TB treatment outcomes. There were some interesting findings.

First, from 2005 to 2013 there was a gradual scale-up of townships implementing collaborative activities focused on HIV testing and providing care and treatment for HIV-associated TB patients. Since 2013, however, there has been a dramatic expansion and all townships implemented collaborative activities by 2016. This increase in TB-HIV collaborative activities, along with the shifting in responsibilities for HIV testing and initiation of CPT and ART, coincided with the implementation of GFATM performance-based financing, ${ }^{12}$ which emphasizes the crucial importance of targeted international support if large-scale change in countries is desired. Since 2002, the GFATM has provided more than three-quarters of all international financing for global TB control, disbursing US\$4.7 billion in more than 100 countries. $^{14}$ In 2013 the GFATM launched its new funding model, which seeks to enhance a synergistic response to HIV-associated TB epidemics and build the necessary resilient and sustainable health systems that underpin TB-HIV collaborative activities. ${ }^{14}$ Myanmar is an example of what can be done if adequate resources are made available and there is the political will to move forward.

Second, with GFATM support there has been a remarkable increase in the numbers and proportions of TB patients in Myanmar tested for HIV, which in 2016 reached $82 \%$. HIV testing (with the important logistics of ensuring uninterrupted supplies of test kits)

Table 3. Demographic and clinical characteristics of patients registered with HIV-associated TB in the Mandalay Region, Myanmar, 2015, in relation to unfavourable treatment outcomes

\begin{tabular}{|c|c|c|c|c|c|}
\hline Total & 815 & $191(23)$ & - & - & - \\
\hline \multicolumn{6}{|l|}{ Age group in years } \\
\hline $15-44$ & 606 & $131(22)$ & Ref & 1 & Ref \\
\hline$\geq 45$ & 146 & $50(34)$ & $1.6(1.2$ to 2.1$)$ & 1.6 (1.2 to 2.1$)$ & $<0.001$ \\
\hline Female & 251 & $68(27)$ & $1.2(0.9$ to 1.6$)$ & 1 (0.7 to 1.3$)$ & 0.8 \\
\hline \multicolumn{6}{|l|}{ Category of TB } \\
\hline New & 718 & $166(23)$ & Ref & 1 & Ref \\
\hline Relapse & 86 & $25(29)$ & 1.3 (0.9 to 1.8 ) & 0.8 (0.5 to 1.2$)$ & 0.3 \\
\hline Treatment after LTFU & 4 & $0(0)$ & - & - & - \\
\hline Treatment after failure & 7 & $0(0)$ & - & - & - \\
\hline CPT alone & 162 & $81(50)$ & $3.3(2.5$ to 4.1$)$ & 1.2 (0.9 to 1.6$)$ & 0.2 \\
\hline ART alone & 34 & $4(12)$ & $0.8(0.3$ to 2.0$)$ & 1.6 (1.0 to 2.6 ) & 0.1 \\
\hline CPT and ART together & 598 & $92(15)$ & Ref & 1 & Ref \\
\hline No adjunctive treatment & 14 & $9(64)$ & $4.2(2.7$ to 6.4$)$ & 1.9 (1.0 to 3.6$)$ & 0.04 \\
\hline No information & 7 & $5(71)$ & $4.6(2.8$ to 7.7$)$ & 2.1 (1.0 to 4.7$)$ & 0.1 \\
\hline
\end{tabular}


Table 4. Demographic and clinical characteristics of patients registered with HIV-associated TB in the Mandalay Region, Myanmar, 2015, in relation to death

\begin{tabular}{|c|c|c|c|c|c|}
\hline Total & 815 & $146(18)$ & & & \\
\hline \multicolumn{6}{|l|}{ Age group in years } \\
\hline $15-44$ & 606 & $97(16)$ & Ref & 1 & Ref \\
\hline$\geq 45$ & 146 & $40(27)$ & 1.7 (1.2 to 2.4 ) & 1.6 (1.2 to 2.2 ) & $<0.01$ \\
\hline Female & 251 & $50(20)$ & 1.2 (0.9 to 1.6$)$ & 1.2 (0.9 to 1.6$)$ & 0.3 \\
\hline \multicolumn{6}{|l|}{ Category of TB } \\
\hline New & 718 & $129(18)$ & Ref & 1 & Ref \\
\hline Relapse & 86 & $17(20)$ & 1.1 (0.7 to 1.7 ) & 1.2 (0.8 to 1.9 ) & 0.4 \\
\hline Treatment after LTFU & 4 & $0(0)$ & - & - & - \\
\hline Treatment after failure & 7 & $0(0)$ & - & - & - \\
\hline CPT alone & 162 & $67(41)$ & 3.9 (2.9 to 5.2 ) & 3.7 (2.7 to 4.9 ) & $<0.001$ \\
\hline ART alone & 34 & $3(9)$ & 0.8 (0.3 to 2.5$)$ & $0.9(0.3$ to 2.6$)$ & 0.8 \\
\hline CPT and ART together & 598 & $64(11)$ & Ref & 1 & Ref \\
\hline No adjunctive treatment & 14 & $9(64)$ & 6.0 (3.8 to 9.5 ) & 6.5 (4.2 to 9.9$)$ & $<0.001$ \\
\hline No information & 7 & $3(43)$ & 4.0 (1.6 to 9.7$)$ & $4.2(1.6$ to 10.8$)$ & $<0.01$ \\
\hline
\end{tabular}

has been able to keep pace with the large numbers of HIVassociated TB patients enrolled in care. In 2015, globally about 55\% of TB patients had a documented HIV result, with the level of HIV testing in the 30 high HIV-associated TB burden countries averaging $64 \%,{ }^{1}$ so Myanmar is performing well in this regard. With more than three-quarters of TB patients taking up provider-initiated HIV testing and counselling in the last 3 years, the prevalence of HIV appears to have stabilized at about $10 \%$. This probably presents a true picture of the prevalence of HIV among TB patients in the country, as in previous years, with fewer patients being tested, it is possible that testing was only offered to those suspected of having HIV, biasing the observed prevalence upwards.

Third, while the uptake of CPT in HIV-associated TB patients has been reasonable at between 70 and $90 \%$ in the last 4 years, the same cannot be said for ART, where uptake has been on average below or just above $50 \%$. In 2015 , globally about $78 \%$ of notified HIV-associated TB patients were reported to be on ART, with the proportion being $80 \%$ in high HIV-associated TB burden countries. ${ }^{1}$ It is possible that in Myanmar there is a weakness of recording and reporting of CPT and ART uptake at the township level, as our regional study in Mandalay, which focused on individual patients, found CPT and ART uptakes to be greater than $90 \%$ and $75 \%$, respectively.

Fourth, in the Mandalay study, HIV-associated TB patients had generally good treatment success, exceeding the global target of
$75 \%$. The overall unfavourable treatment outcome was $23 \%$, which was predominately due to a high mortality approaching $20 \%$. The low loss to follow-up of $2 \%$ bears testimony to good tracking of patients during treatment. The main factors associated with both an unfavourable treatment outcome and death were older age ( $\geq 45$ years) and being on CPT alone or on neither CPT or ART.

The findings in Mandalay on age concur with previous studies documenting worse treatment outcomes in older adults. ${ }^{15,16}$ There are various possible explanations. When diagnostic resources are limited, it is possible that older patients have a higher prevalence of chronic lung disease with various aetiologies that are potentially misdiagnosed as PTB and without specific treatment mortality may be higher with these chronic respiratory conditions. Older patients may also have other comorbidities that compromise TB treatment outcomes. Diabetes mellitus is known to adversely affect TB treatment outcomes by increasing the risk of death or treatment failure in patients with dual disease, ${ }^{17}$ and particularly so if patients have both diabetes and are current smokers. ${ }^{18}$ Large studies conducted in China and India, using fasting blood glucose as the diagnostic method, found the diabetes prevalence in TB patients to be between 12 and 13\%, with a significant proportion having diabetes newly diagnosed as a result of active screening initiatives. $^{19,20}$

While CPT reduces mortality risk in people living with HIV and results in fewer episodes of malaria and increased overall life 
expectancy, ${ }^{21}$ its effects are limited when used on its own, as shown in our current study. The key intervention is ART, preferably used in conjunction with CPT, and this has been shown in clinical trials to greatly reduce mortality, especially if used early in the course of anti-TB treatment. ${ }^{22,23}$ This is in agreement with another recent study in Myanmar that found excellent treatment outcomes among HIV-associated TB patients started on ART. ${ }^{24}$

The strengths of this study were the national sample assessed over a 12-year period and the large number of patients enrolled in the Mandalay Region in 2015. We also adhered to Strengthening the Reporting of Observational Studies in Epidemiology guidelines for the conduct and reporting of this observational study. ${ }^{25}$ Limitations relate to the retrospective operational nature of the study, which relied on a mix of paper-based and electronic national aggregate reports for the national data and three different sources for the individual data. For the national reports, we were unable to obtain specific township CPT data for 3 years from 2008 to 2010, and we do not know whether the low ART uptake was due to incomplete recording and reporting or failure of implementation. For the individual data, we also did not collect information about when CPT or ART was started (either before or after the start of anti-TB treatment) or about drug resistance, yet both of these may be determinants of treatment outcome.

There are some programmatic implications from this study. First, with collaborative activities to reduce the double burden of TB and HIV now expanded to all townships in the country, it will be important to improve on quality and obtain maximum benefit by ensuring as close to 100\% HIV testing of registered TB patients as possible, and for those who are HIV positive, 100\% uptake of CPT and ART. This will require sustained funding, good working relationships between the NAP and NTP at all levels, uninterrupted supplies of consumables and drugs, prompt initiation of ART after starting anti-TB treatment and accurate, comprehensive recording and reporting of HIV parameters in TB treatment cards and registers.

Second, the growing burden of non-communicable diseases in patients with HIV and TB needs to be recognized and addressed if mortality is to be reduced. ${ }^{26}$ Screening TB patients for diabetes, which has already started in a phased approach in Myanmar, should be evaluated and, if deemed feasible and cost effective, scaled up as recommended by the WHO and the International Union Against Tuberculosis and Lung Disease. ${ }^{27}$ Decisions will need to be made about whether to screen all patients or those most at risk based on, for example, age, body mass index or smoking status.

Finally, Myanmar needs to fully embrace the other important TB-HIV collaborative activity of reducing the double burden of TB and HIV by getting all people infected with HIV on ART, as recommended in the recent guidance by the $\mathrm{WHO},{ }^{28}$ and considering the additional use of isoniazid preventive therapy, which further reduces the risk of $\mathrm{TB}^{29}{ }^{29}$ There is growing evidence that at the population level, the scale-up of ART with high levels of coverage is associated with a significant decline in the national burden of TB, both for HIV-infected and non-infected communities. ${ }^{30}$

In conclusion, in the 12 years from 2005 to 2016, Myanmar has expanded TB-HIV collaborative activities to its townships to reduce the double burden of TB and HIV, and there has been a significant increase in registered TB patient numbers, HIV testing uptake and initiation of CPT and ART in HIV-associated TB patients since 2013.
Although at the national level ART uptake in HIV-associated TB patients is about $50 \%$, this is higher (at $>75 \%$ ) in the Mandalay Region and is associated with good treatment outcomes. Risk factors for unfavourable outcomes and death include age $\geq 45$ years and no adjunctive treatment with CPT and/or ART. These findings have programmatic implications for moving forward.

Authors' contributions: PT and STA conceived the study. PT, ADH, HDS, SS and KTW designed the study protocol and all authors read and approved the study protocol. PT collected the data. All authors contributed to analysing and interpreting the data. PT, ADH, HDS, SS and KTW drafted the manuscript and all authors critically revised the manuscript for intellectual content. All authors read and approved the final manuscript. PT and KTW are guarantors of the paper.

Acknowledgements: This research was conducted through the Structured Operational Research and Training Initiative (SORT IT), a global partnership led by the Special Programme for Research and Training in Tropical Diseases at the World Health Organization (WHO/TDR). The model is based on a course developed jointly by the International Union Against Tuberculosis and Lung Disease (The Union) and Medécins sans Frontières (MSF/Doctors Without Borders). The specific SORT IT programme that resulted in this publication was jointly organized and implemented by the Centre for Operational Research, The Union, Paris, France; the Department of Medical Research, Ministry of Health and Sports, Myanmar; the Department of Public Health, Ministry of Health and Sports, Myanmar; The Union Country Office, Mandalay, Myanmar; The Union South-East Asia Office, New Delhi, India; the Operational Research Unit (LUXOR), MSF Brussels Operational Center, Luxembourg and Burnet Institute, Melbourne, VIC, Australia.

Funding: The training programme within which this paper was developed and the open access publication costs were funded by the Department for International Development (DFID), London, UK, and La Fondation Veuve Emile Metz-Tesch (Luxembourg). The funders had no role in the study design, data collection and analysis, decision to publish or preparation of the manuscript.

Competing interests: None declared.

Ethical approval: Ethics approval in Myanmar was obtained from the Ethics Review Committee on Medical Research involving Human Subjects, Department of Medical Research, Ministry of Health and Sports. Ethics approval was also obtained from the Union Ethics Advisory Group, International Union against Tuberculosis and Lung Disease, Paris, France. Informed patient consent was not required since aggregate or secondary programmatic data were used.

\section{References}

1 World Health Organization. Global tuberculosis report 2016. WHO/ HTM/TB/2016.23. Geneva: World Health Organization; 2016.

2 Diul MY, Maher D, Harries AD. Tuberculosis case fatality rates in HIV prevalence populations in sub-Saharan Africa. AIDS 2001;15(2): 143-52.

3 World Health Organization. Interim policy on collaborative TB/HIV activities. WHO/HTM/TB/2004.330.Geneva: World Health Organization; 2004.

4 World Health Organization. WHO policy on collaborative TB/HIV activities. Guidelines for national programmes and other stakeholders. WHO/HTM/TB 2012.1 or WHO/HIV/2012.1. Geneva: World Health Organization; 2012. 
5 Harries AD, Zachariah R, Corbett EL et al. The HIV-associated tuberculosis epidemic — when will we act? Lancet 2010;375(9729):1906-19.

6 National AIDS Programme. HIV sero-sentinel surveillance survey in Myanmar. Myanmar: Ministry of Health; 2014.

7 Thandar Lwin TL, Moe Zaw MZ, Khin Ohnmar San KOS et al. Routine provider-initiated HIV counselling and testing of adult TB patients in Myanmar [abstract PC-100300-15]. Union World Lung Health Conference, Berlin, 11-15 November 2010.

8 The 2014 Myanmar population and housing census: The Union Report. Department of Population, Ministry of Immigration and Population, Myanmar. May 2015.

9 World Health Organization. Treatment of tuberculosis guidelines, 4th ed. WHO/HTM/TB/2009.420. Geneva: World Health Organization; 2010.

10 National AIDS Programme. Guidelines for the clinical management of HIV infection in adults and adolescents in Myanmar, 3rd ed. Myanmar: Department of Health, Ministry of Health; 2011.

11 National AIDS Programme. Guidelines for the clinical management of HIV infection in Myanmar, 4th ed. Myanmar: Department of Health, Ministry of Health; 2014.

12 National Tuberculosis Programme. Annual report (2013). Myanmar: Ministry of Health; December 2014.

13 National Tuberculosis Programme and National AIDS Programme. Guidelines for the programmatic management of TB/HIV in Myanmar. Myanmar: Ministry of Health; December 2015.

14 Kunii O, Yassin MA, Wandwalo E. Investing to end epidemics: the role of the Global Fund to control TB by 2030. Trans R Soc Trop Med Hyg 2016;110(3):153-4.

15 Ananthakrishnan R, Kumar K, Ganesh M et al. The profile and treatment outcomes of the older (aged 60 years and above) tuberculosis patients in Tamil Nadu, South India. PLoS One 2013;8(7):e67288.

16 Tagaro M, Harries AD, Kool B et al. Tuberculosis case burden and treatment outcomes in children, adults and older adults, Vanuatu, 2007-2011. Public Health Action 2014;4(Suppl 1):S14-18.

17 Baker MA, Harries AD, Jeon CY et al. The impact of diabetes on tuberculosis treatment outcomes: a systematic review. BMC Med 2011;9:81.

18 Reed GW, Choi H, Lee SY et al. Impact of diabetes and smoking on mortality in tuberculosis. PLoS One 2013;8(2):e58044.
19 Lin Y, Li L, Mi F et al. Screening of patients with diabetes mellitus for tuberculosis in China. Trop Med Int Health 2012;17(10):1302-8.

20 India Diabetes Mellitus-Tuberculosis Study Group. Screening of patients with diabetes mellitus for tuberculosis in India. Trop Med Int Health 2013;18(5):646-54.

21 Suthar AB, Vitoria MA, Nagata JM et al. Co-trimoxazole prophylaxis in adults, including pregnant women with HIV: a systematic review and meta-analysis. Lancet HIV 2015;2(4):e137-50.

22 Blanc FX, Sok T, Laureillard D et al. Earlier versus later start of antiretroviral therapy in HIV-infected adults with tuberculosis. N Engl J Med 2011;365:1471-81.

23 Havlir DV, Kendall MA, Ive P et al. Timing of antiretroviral therapy for HIV-1 infection and tuberculosis. N Engl J Med 2011;365:1482-91.

24 Thi AM, Shewade HD, Kyaw NTT et al. Timing of antiretroviral therapy and TB treatment outcomes in patients with TB-HIV in Myanmar. Public Health Action 2016;6(2):111-7.

25 Von Elm E, Altman DG, Egger $M$ et al. The Strengthening the Reporting of Observational Studies in Epidemiology (STROBE) statement: guidelines for reporting observational studies. Lancet 2007; 370:1453-7.

26 Hyle EP, Naidoo K, Su AE et al. HIV, tuberculosis, and noncommunicable diseases: what is known about the costs, effects, and costeffectiveness of integrated care? J Acquir Immune Defic Syndr 2014; 67(Suppl 1):S87-95.

27 World Health Organization and the International Union Against Tuberculosis and Lung Disease. Collaborative framework for care and control of tuberculosis and diabetes. WHO/HTM/TB/2011.15. Geneva: World Health Organization; 2011.

28 World Health Organization. Consolidated guidelines on the use of antiretroviral drugs for treating and preventing HIV infection. Recommendations for a public health approach, 2nd ed. Geneva: World Health Organization; 2016.

29 Rangaka MX, Wilkinson RJ, Boulle A et al. Isoniazid plus antiretroviral therapy to prevent tuberculosis: a randomised double-blind, placebo-controlled trial. Lancet 2014;384(9944):682-90.

30 Kanyerere H, Girma B, Mpunga J et al. Scale-up of ART in Malawi has reduced case notification rates in HIV-positive and HIV-negative tuberculosis. Public Health Action 2016;6:247-51. 